\title{
Sensor and Actuator Fault Reconstruction for Nonlinear System XIONG Yi
}

Hunan Railway Professional Technology College, Zhuzhou, 412001

Keywords: coordinate transformation, robust fault diagnosis, fault reconstruction, sliding mode observer

\begin{abstract}
This paper presents a fault reconstruction scheme based on sliding mode observers for sensor and actuator faults detection and isolation for a class of uncertain nonlinear systems. A coordinate transformation is first designed for the output equation of the system followed by a firstorder low-pass filter in order to convert the sensor faults into equivalent actuator faults. The original system then is transformed into three subsystems through linear transformation.
\end{abstract}

\section{Introduction}

System faults and unknown input disturbances are inevitable during operation of a complicated power system. With regard to physical position, faults can be classified into actuator and sensor faults. Faults can damage the normal system operation and make the system unstable, therefore, the fault detection and isolation (FDI) technique plays an important role in system operation. In the past decades, the study on FDI made great progress, especially for the model-based fault detection [1-3]. Various approaches have been proposed to solve FDI problems, such as differential geometry method, self-adaptive control method, and sliding mode observer technique. Moreover, research on fault tolerance control and stability analysis considerably promoted the FDI [4-6]. The power system used in this study inevitably suffers from unknown input disturbances and potential system faults during operation. Therefore, the sliding mode control technique, an effective method with has high robustness and insensitivity to system uncertainty, has been extensively employed in the study for FDI .

\section{Problem description}

A nonlinear system with actuator and sensor faults is given by

$$
\left\{\begin{array}{c}
\dot{x}(t)=A x+\Phi(x, u)+E f_{a}(t)+D d(t)+B u(t) \\
y(t)=C x(t)+F_{s} f_{s}(t)
\end{array}\right.
$$

where $x \in R^{n}, u \in R^{r}$ and $y \in R^{p}$ denote, respectively, the state variables, inputs and outputs. The nonlinear continuous term $\Phi(x, u) \in R^{n}$ is assumed to be known. The unknown nonlinear term $d(t) \in R^{q}$ models the lumped uncertainties and disturbances experienced by the system, which is assumed to be bounded, i.e., a positive constant $\gamma_{1}$ exists such that $\|d(t)\| \leq \gamma_{1}$. The unknown nonlinear terms $f_{a}(t) \in R^{q}$ and $f_{s}(t) \in R^{m}$ denote, respectively, actuator faults and sensor faults, which are also bounded, i.e., two constant $\gamma_{2}$ and $\gamma_{3}$ exist such that $\left\|f_{a}(t)\right\| \leq \gamma_{2}$ and $\left\|f_{s}(t)\right\| \leq \gamma_{3} . A \in R^{n \times n}, B \in R^{n \times r}, C \in R^{p \times n}, D \in R^{n \times q}, E \in R^{n \times q}$ and $F_{s} \in R^{p \times m}$ are known constant matrices with $n>p>q+m$.

For the output signal $y \in R^{p}$ of system (1), a transformation matrix $S_{0} \in R^{p \times p}$ exists such that the following equation could be obtained:

we obtain

$$
S_{0}\left[\begin{array}{ll}
C & F_{s}
\end{array}\right]=\left[\begin{array}{ll}
C_{1} & 0 \\
C_{2} & F
\end{array}\right]
$$




$$
S_{0} y=\left\{\begin{array}{c}
y_{1}=C_{1} x \\
y_{2}=C_{2} x+F f_{s}
\end{array}\right.
$$

where $y_{1} \in R^{p-m}, y_{2} \in R^{m}, C_{1} \in R^{(p-m) \times n}, C_{2} \in R^{m \times n}$, and $F \in R^{m \times m}$ is a nonsingular constant matrix. Partition of the outputs $y_{1}$ and $y_{2}$ represents the fault free sensor signals and the potentially faulty sensor signals, respectively.

A new status variable $z_{a} \in R^{m}$ is then designed as the first-order low-pass filter of the output signal $y_{2}$

$$
\dot{z}_{a}=A_{s} z_{a}+B_{s} y_{2}
$$

where $A_{s} \in R^{m \times m}$, and $B_{s} \in R^{m \times m}$ are filter matrices to be determined and $B_{s}$ is a constant matrix with full rank.

The following equation could be obtained by transforming the output signal $y_{2}$ of Eq. (3) to Eq. (4)

$$
\dot{z}_{a}=A_{s} z_{a}+B_{s} C_{2} X+B_{s} F f_{s}
$$

Eq. (5) and System (1) are then combined into a new system equation.

$$
\left\{\begin{array}{c}
{\left[\begin{array}{c}
\dot{x} \\
\dot{z}_{a}
\end{array}\right]=\left[\begin{array}{cc}
A & 0 \\
B_{s} C_{2} & A_{s}
\end{array}\right]\left[\begin{array}{c}
x \\
z_{a}
\end{array}\right]+\left[\begin{array}{c}
\Phi(x, u) \\
0
\end{array}\right]+\left[\begin{array}{c}
E \\
0
\end{array}\right] f_{a}(t)+\left[\begin{array}{c}
0 \\
B_{s} F
\end{array}\right] f_{s}(t)+\left[\begin{array}{c}
D \\
0
\end{array}\right] d(t)+\left[\begin{array}{c}
B \\
0
\end{array}\right] u(t)} \\
{\left[\begin{array}{c}
y_{1} \\
z_{a}
\end{array}\right]=\left[\begin{array}{cc}
C_{1} & 0 \\
0 & I_{m}
\end{array}\right]\left[\begin{array}{c}
x \\
z_{a}
\end{array}\right]}
\end{array}\right.
$$

A set of new status variables and the corresponding matrix are then designed as follows:

$$
\bar{x}=\left[\begin{array}{c}
x \\
z_{a}
\end{array}\right], \bar{y}=\left[\begin{array}{l}
y_{1} \\
z_{a}
\end{array}\right]=\left[\begin{array}{l}
y_{1} \\
y_{a}
\end{array}\right], \bar{A}=\left[\begin{array}{cc}
A & 0 \\
B_{s} C_{2} & A_{s}
\end{array}\right], \bar{C}=\left[\begin{array}{cc}
C_{1} & 0 \\
0 & I_{m}
\end{array}\right]
$$

where $\bar{x} \in R^{n+m}, \bar{y} \in R^{p}, \bar{A} \in R^{(n+m) \times(n+m)}$ and $\bar{C} \in R^{p \times n}$.

Eq.(6) could be converted into the following two subsystems

$$
\begin{aligned}
& \left\{\begin{array}{c}
\dot{x}(t)=A x+\Phi(x, u)+E f_{a}(t)+D d(t)+B u(t) \\
y_{1}(t)=C_{1} x(t)
\end{array}\right. \\
& \left\{\begin{array}{c}
\dot{z}_{a}=A_{s} z_{a}+B_{s} C_{2} x+B_{s} F f_{s} \\
y_{a}=z_{a}
\end{array}\right.
\end{aligned}
$$

\section{Fault reconstruction and disturbance estimation}

When the system reaches the sliding mode surface, $s_{i}=\dot{s}_{i}=0(i=1,2,3)$ according to the sliding mode equivalent principle. The following equation is obtained:

$$
\begin{gathered}
F_{1} e_{v 1}=F_{1} \dot{e}_{v 1}=F_{1} C_{11} \dot{e}_{1}=0 \\
F_{2} e_{v 2}=F_{2} \dot{e}_{v 2}=F_{2} C_{22} \dot{e}_{2}=0 \\
e_{a}=\dot{e}_{a}=0
\end{gathered}
$$

From lemma $1, e_{1}$ and $e_{2}$ will approach zero in some finite time and $\Phi(z, u)=\Phi(\hat{z}, u)$.

(1) The reconstruction of actuator fault $f_{\mathrm{a}}(t)$

We obtain the following:

$$
\begin{gathered}
F_{1} C_{11}\left(\left(\bar{A}_{11}-L_{1} C_{11}\right) e_{1}(t)+\bar{A}_{12} e_{2}(t)+\bar{\Phi}_{1}(z, u)-\bar{\Phi}_{1}(\hat{z}, u)+\bar{E}_{1} f_{a}(t)-\bar{E}_{1} r_{1}(t)\right)=0 \\
f_{a}(t) \approx r_{1}(t)=\rho_{1} \operatorname{sgn}\left(F_{1} e_{v 1}\right)
\end{gathered}
$$

(2) The reconstruction of sensor fault $f_{\mathrm{s}}(t)$

The following equation could be obtained:

$$
A_{1} e_{1}(t)+A_{2} e_{2}(t)+A_{s} e_{a}(t)+B_{s} F f_{s}(t)-r_{3}(t)=0
$$


$F \in R^{m \times m}$ is a nonsingular matrix and $B_{s} \in R^{m \times m}$ is a full rank matrix to be designed. Therefore, a matrix $B_{\mathrm{s}}$ exists to make $B_{\mathrm{s}} F$ to be a nonsingular matrix, then

$$
f_{s}(t) \approx\left(B_{s} F\right)^{-1} r_{3}(t)=\left(B_{s} F\right)^{-1} \rho_{3} \operatorname{sgn}\left(e_{a}\right)
$$

(3) The estimation of the unknown input disturbance $d(t)$

we obtain the following:

$$
\begin{gathered}
F_{2} C_{22}\left(\left(\bar{A}_{22}-L_{2} \bar{C}_{22}\right) e_{2}(t)+\bar{A}_{21} e_{1}(t)+\bar{\Phi}_{2}(x, u)-\bar{\Phi}_{2}(\hat{x}, u)+\bar{E}_{2} f_{a}(t)-\bar{E}_{2} r_{2}(t)+\bar{D}_{2} d(t)\right)=0 \\
d(t) \approx \bar{D}_{2}^{-1}\left(\bar{E}_{2} r_{2}(t)-\bar{E}_{2} f_{a}(t)\right)=\bar{D}_{2}^{-1} \bar{E}_{2}\left(\rho_{2} \operatorname{sgn}\left(F_{2} e_{v 2}\right)-\rho_{1} \operatorname{sgn}\left(F_{1} e_{v 1}\right)\right) \\
\operatorname{sgn}\left(F_{1} e_{v 1}\right)=\frac{F_{1} e_{v 1}}{\left|F_{1} e_{v 1}\right|+\sigma_{1}}, \operatorname{sgn}\left(F_{2} e_{v 2}\right)=\frac{F_{2} e_{v 2}}{\left|F_{2} e_{v 2}\right|+\sigma_{2}}, \operatorname{sgn}\left(e_{a}\right)=\frac{e_{a}}{\left|e_{a}\right|+\sigma_{3}}
\end{gathered}
$$

where $\sigma_{1}, \sigma_{2}, \sigma_{3}$ are the three minor positive constants.

\section{Simulation example}

Consider a single-link robotic arm with a revolute elastic joint rotating in a vertical plane whose motion equations are as follows:

$$
\left\{\begin{array}{c}
J_{1} \ddot{q}_{1}+F_{n} \dot{q}_{1}+k\left(q_{1}-q_{2}\right)+m g l \sin q_{1}=0 \\
J_{m} \ddot{q}_{2}+F_{m} \dot{q}_{2}-k\left(q_{1}-q_{2}\right)=u
\end{array}\right.
$$

where $q_{1}$ and $q_{2}$ are the link displacement and the rotor displacement, respectively. The link intertia $J_{1}$, motor rotor inertia $J_{\mathrm{m}}$, elastic constant $k$, link mass $m$, gravity $g$, connecting rod length $l$, and viscous friction coefficients $F_{1}, F_{\mathrm{m}}$ are all positive constant parameters. The control $u$ is the torque delivered by the motor. The robot parameters are include $k=2 \mathrm{Nm} / \mathrm{rad}, F_{\mathrm{m}}=1$, $F_{1}=0.5 \mathrm{Nm} /(\mathrm{rad} / \mathrm{s}), \quad J_{\mathrm{m}}=1 \mathrm{Nm}^{2}, \quad J_{1}=2 \mathrm{Nm}^{2}, \quad m=0.15 \mathrm{~kg}, \quad g=9.8 \mathrm{~m} / \mathrm{s}^{2}, \quad$ and $\quad l=0.3 \mathrm{~m}$. The input $u=\sin (5 t)+4 \sin (20 t)$.

The state variables are defined as $x_{1}=q_{1}, x_{2}=\dot{q}_{1}, x_{3}=q_{2}$, and $x_{4}=\dot{q}_{2}$. The robot parameters in practice vary as time changes with handling different objects. Such changing parameters are defined as unknown input disturbances. Assuming that there exists an actuator fault $f_{a}(t)$ and a sensor fault $f_{s}(t)$ on the single-articulation robot, the system equation as shown Eq. (1) can be obtained.

Correspond (1), the parameter matrixes are

$$
\begin{gathered}
A=\left[\begin{array}{cccc}
0 & 1 & 0 & 0 \\
\frac{-k}{J_{1}} & \frac{-F_{1}}{J_{1}} & \frac{k}{J_{1}} & 0 \\
0 & 0 & 0 & 1 \\
\frac{k}{J_{m}} & 0 & \frac{-k}{J_{m}} & \frac{-F_{m}}{J_{m}}
\end{array}\right]=\left[\begin{array}{cccc}
0 & 1 & 0 & 0 \\
-1 & -0.25 & 1 & 0 \\
0 & 0 & 0 & 1 \\
2 & 0 & -2 & -1
\end{array}\right] \\
f(x, u, t)=\left[\begin{array}{c}
0 \\
-0.2205 \sin x_{1} \\
0 \\
0
\end{array}\right], \quad E=\left[\begin{array}{l}
3 \\
4 \\
4 \\
1
\end{array}\right], \quad D=\left[\begin{array}{l}
0 \\
1 \\
0 \\
\frac{1}{2}
\end{array}\right] \\
B=\left[\begin{array}{l}
0 \\
0 \\
0 \\
1
\end{array}\right], \quad C=\left[\begin{array}{llll}
1 & 0 & 0 & 0 \\
0 & 0 & 1 & 0 \\
0 & 1 & 0 & 1 \\
0 & 0 & 0 & 1
\end{array}\right], \quad F=\left[\begin{array}{l}
0 \\
0 \\
1 \\
0
\end{array}\right]
\end{gathered}
$$

The transformational matrix $T$ 


$$
T=\left[\begin{array}{cccc}
1 & 0 & 0 & 0 \\
0 & 1 & 0 & -2 \\
0 & 0 & 1 & 0 \\
0 & 0 & 0 & 1
\end{array}\right]
$$

then

The transformational matrices $S_{0}, S$

$$
T E=\left[\begin{array}{l}
3 \\
2 \\
4 \\
1
\end{array}\right], T D=\left[\begin{array}{l}
0 \\
0 \\
0 \\
1
\end{array}\right]
$$

$$
S_{0}=\left[\begin{array}{llll}
1 & 0 & 0 & 0 \\
0 & 1 & 0 & 0 \\
0 & 0 & 0 & 1 \\
0 & 0 & 1 & 0
\end{array}\right], \quad S=\left[\begin{array}{lll}
1 & 0 & 0 \\
0 & 1 & 0 \\
0 & 0 & 1
\end{array}\right]
$$

Design the matrices as $P_{1}=\left[\begin{array}{ccc}\frac{1}{3} & 0 & 0 \\ 0 & \frac{1}{2} & -\frac{1}{4} \\ 0 & -\frac{1}{4} & \frac{3}{8}\end{array}\right], L_{1}=\left[\begin{array}{cc}4 & 2 \\ -8 & 1 \\ 4 & 1\end{array}\right], \quad P_{2}=[1], L_{2}=[2], \quad F_{1}=\left[\begin{array}{ll}1 & 1\end{array}\right]$, $F_{2}=[1], A_{s}=[-2]$ and $B_{s}=[2]$.

The actuator fault reconstruction algorithm

$$
\hat{f}_{a}(t)=r_{1}(t)=\rho_{1} \operatorname{sgn}\left(F_{1} e_{v 1}\right)=\rho_{1} \frac{e_{1}+e_{3}}{\left|e_{1}+e_{3}\right|+\sigma_{1}}
$$

The sensor fault reconstruction algorithm

$$
\hat{f}_{s}(t)=\left(B_{s} F\right)^{-1} \rho_{3} \operatorname{sgn}\left(e_{a}\right)=0.5\left(\rho_{3} \frac{e_{a 1}}{\left|e_{a 1}\right|+\sigma_{3}}\right)
$$

The estimation of the unknown input disturbance

$$
\hat{d}(t)=\bar{D}_{2}^{-1} \bar{E}_{2}\left(\rho_{2} \operatorname{sgn}\left(F_{2} e_{v 2}\right)-\rho_{1} \operatorname{sgn}\left(F_{1} e_{v 1}\right)\right)=\rho_{2} \frac{e_{4}}{\left|e_{4}\right|+\sigma_{2}}-\rho_{1} \frac{e_{1}+e_{3}}{\left|e_{1}+e_{3}\right|+\sigma_{1}}
$$

The observer parameters are chosen as $\rho_{1}=32, \rho_{2}=60, \rho_{3}=20, \sigma_{1}=0.1, \sigma_{2}=0.02$, and $\sigma_{3}=0.01$. Two overlapped sine signals are used to simulate the incipient fault for actuator fault $f_{a}(t)$, where $f_{a}=2 \sin 40 t+2 \sin 5 t$. The sensor fault $f_{s}(t)$ is simulated by a white noise with amplitude of 3 and sample period of $0.02 \mathrm{~s}$, which is the combination of abrupt fault and intermittent fault. The unknown input disturbance $d(t)$ is simulated by a sinusoidal, which is $d(t)=4 \sin 20 t$. The initial value of state variable $x$ in the simulation example is chosen as $0,0,-3$, and -2 . The simulation results are as follows: 

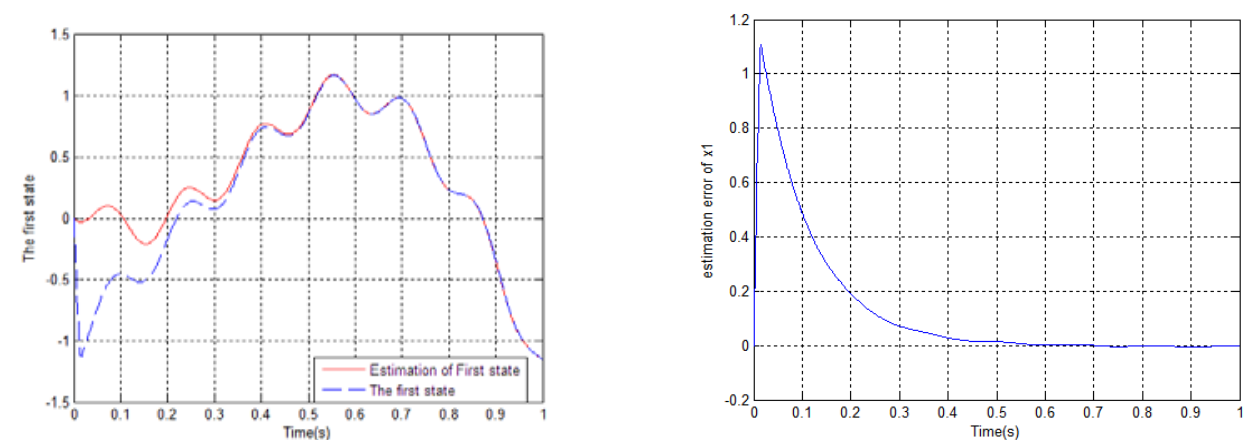

Fig. 1. The first state $x_{1}$ and its estimation $\hat{x}_{1}$

Fig. 2. The estimation error $e_{1}$

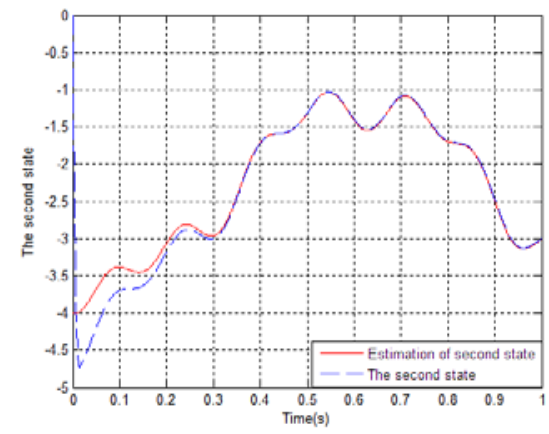

Fig. 3. The second state $x_{2}$ and its estimation $\hat{x}_{2}$

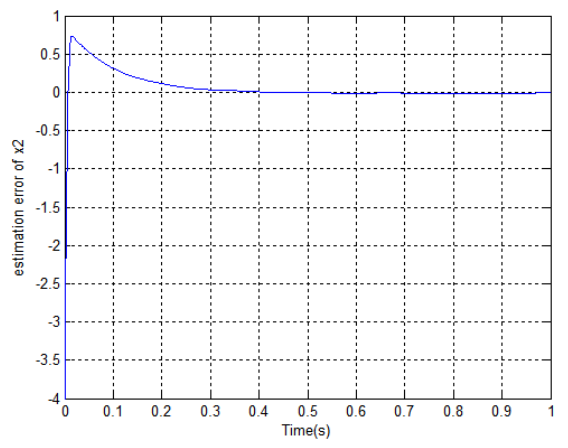

Fig. 4. The estimation error $e_{2}$

Figures 1, 3, show the estimation results of the four state vectors, the corresponding estimation errors are shown on Figures 2, 4,. The results imply that the observers converge quickly, which lay the foundation for fault reconstruction.

\section{Conclusions}

In this paper, a type of nonlinear system with actuator faults, sensor faults, and unknown input disturbances is studied. A fault reconstruction method of nonlinear system is presented.

\section{References}

[1] J.J. Gertler,"Survey of model-based faults detection and isolation in complex plant," IEEE Control Syst.Mag.,vol.8, pp.3-11,1988.

[2] S. Simani, C. Fantuzzi, and R.J. Patton,"Model-based fault diagnosis in dynamic systems using identification techniques," Springer-Verlag,London,2002.

[3] R.J. Patton, R. Clark, and R.N. Clark,"Issues of fault diagnosis for dynamic systems," SpringerVerlag,Berlin Heidelberg,New York,2000.

[4] X.J. Meng and G.H. Yang,"Nonlinear fault tolerant control for polynomial systems with input saturation," The Control and Decision Conference, Guiyang, China, pp.3918-3923,2013.

[5] H.G. Zhang, Z.S. Wang, and D.R. Liu,"A Comprehensive Review of Stability Analysis of Continuous-Time Recurrent Neural Networks," IEEE Transactions on Neural Networks and Learning Systems,vol.25, pp.1229-1262,2014.

[6] X.S. Si and D.H. Zhou,"A Generalized Result for Degradation Model-Based Reliability Estimation," IEEE Transactions on Automation Science and Engineering, vol.11,pp.632$637,2013$. 